\title{
Significant Association of Catechol-O-Methyltransferase (COMT) Haplotypes with Nicotine Dependence in Male and Female Smokers of Two Ethnic Populations
}

\author{
Joke Beuten', Thomas J Payne ${ }^{2}$, Jennie Z Ma' and Ming D Li*, \\ 'Program in Genomics and Bioinformatics on Drug Addiction, Department of Psychiatry, The University of Texas Health Science Center at \\ San Antonio, San Antonio, TX, USA; ${ }^{2}$ The ACT Tobacco Center, The University of Mississippi School of Dentistry, Jackson, MS, USA
}

\begin{abstract}
The catechol-O-methyltransferase (COMT) gene plays a prominent role in dopaminergic circuits central to drug reward. Allelic variants within the COMT gene are therefore potential candidates for examining interindividual differences in vulnerability to nicotine dependence (ND). We analyzed five single nucleotide polymorphisms (SNPs), including the Val/Met variant (rs4680), which results in a three- to fourfold difference in enzyme activity within COMT, for association with the three ND measures, SQ, HSI, and FTND, in 602 nuclear families of African-American (AA) or European-American (EA) origin. The Val/Met variant showed a significant association with the three ND measures in the pooled and EA samples and with FTND in the AA sample. Haplotype analysis revealed a major protective A-G-T haplotype (frequency 23.6\%) for rs740603-rs4680-rs 174699 in the AA sample (minimum $Z=-3.35 ; P=0.0005$ for FTND), a major protective T-G-T haplotype (frequency 15.2\%; minimum $Z=-2.92 ; P=0.003$ for FTND) in the EA sample, and a high-risk C-A-T haplotype (frequency 16.9\%; minimum $Z=3.16 ; P=0.002$ for SQ) in the AA sample for rs93327I-rs4680-rs I 74699. Furthermore, we found that the significant haplotypes within COMT were gender-specific and the significantly associated A-G-T is protective in AA females only, whereas T-G-T is protective in EA males only. Moreover, we found a major high-risk T-A-T haplotype (frequency 56.7\%) that showed significant association with the three ND measures in EA males. Further examination of two protective haplotypes, A-G-T in AAs and T-G-T in EAs, indicated that the low COMT enzyme activity Met allele is protective to become nicotine dependent. In summary, our results provide evidence for a role of COMT in the susceptibility to ND and further confirm that its effect is ethnic and gender specific. Neuropsychopharmacology (2006) 31, 675-684. doi:I0.I038/sj.npp. I 300997; published online I4 December 2005
\end{abstract}

Keywords: haplotype; SNP; COMT; association analysis; nicotine dependence; tobacco smoking

\section{INTRODUCTION}

According to a report from the World Health Organization, 1.3 billion people worldwide smoke tobacco (WHO, 2005). With 5 million tobacco-related deaths per year, tobacco use is the leading cause of preventable death in the world today. Although nearly $70 \%$ of smokers in the U.S. report that they are interested in quitting, $<5 \%$ of those who try quitting remain tobacco-free for 12 months (US Department of Health and Human Services, 1988). There is substantial evidence for a genetic component in the vulnerability to nicotine dependence (ND) based on twin and family segregation studies (Sullivan and Kendler, 1999; Li et al, 2003) as well as data from studies with inbred mouse strains

\footnotetext{
*Correspondence: Dr MD Li, Department of Psychiatric Medicine, University of Virginia, 1670 Discovery Drive, Suite 1 I0, Charlottesville, VA 229II, USA, Tel: + I 434243 0566; Fax: + I 434973 730I;

E-mail:ml2km@virginia.edu

Received 7 July 2005; revised 13 October 2005; accepted 3 I October 2005

Online publication: I November 2005 at http://www.acnp.org/citations/ Npp I 0 | 05050436/default.pdf
}

(Hatchell and Collins, 1980; Robinson et al, 1996). However, only limited association studies on candidate genes and/or linkage analyses for susceptibility loci have consistently produced positive findings (for a review, see Li et al, 2004).

Nicotine has been shown to be the primary addictive component of tobacco smoking, stimulating the release of dopamine from neurons in the ventral tegmental area, an action thought to underlie its rewarding effects (Nisell et al, 1994; Pontieri et al, 1996). ND is a complex disorder for which the combined effects of multiple interactive genes, each with a small effect, likely confer additive genetic contributions. The influence of genes on regulatory processes in the human brain is particularly difficult to resolve, given that a functional genetic variant may affect not only the protein coded by the gene in question, but may also have downstream effects, contributing to the overall system response.

In this study, we focus on the catechol-O-methyltransferase (COMT) gene, first cloned by Lundstrom et al (1991). The protein encoded by this gene catalyzes the transfer of a methyl group from S-adenosyl-methionine (SAM) to a hydroxyl group of catecholamines, including the 
neurotransmitters dopamine, epinephrine and norepinephrine, or of catechol estrogen resulting in the degradation of catecholamines (Weinshilboum et al, 1999). COMT is encoded by a single gene with six exons that has been mapped to chromosome 22q11.21 (Grossman et al, 1992). The COMT protein exists in two isoforms resulting from two mRNA transcripts from two promoters (P1 and P2): a soluble form found in the cell cytoplasm (S-COMT; $221 \mathrm{aa}$ ) and a longer, membrane-bound form (MB-COMT; $271 \mathrm{aa})$ (Tenhunen et al, 1994). The MB-COMT is predominantly expressed in the brain, while S-COMT is predominantly expressed in blood and in other tissues, such as liver and kidney (Tenhunen et al, 1994; Lundstrom et al, 1995). Although COMT is expressed widely throughout the brain, its enzymatic activity appears to be particularly important in the prefrontal cortex, where it inactivates dopamine (Garris et al, 1993; Gogos et al, 1998; Matsumoto et al, 2003).

The important role of COMT in the dopamine pathway has further been demonstrated by genetic and pharmacological studies in rodents. For example, COMT-deficient mice exhibit sexually dimorphic and region-specific changes in dopamine levels, notably in the prefrontal cortex (Gogos et al, 1998; Akil et al, 2003). In addition, COMT knockout mice show a number of behavioral and neurological effects, including alterations in anxiety-like behavior (Holmes, 2001). Heterozygous COMT-deficient male mice further exhibit increased aggressive behavior (Gogos et al, 1998), and the more aggressive inbred strains had lower COMT expression levels in the hippocampus relative to the less aggressive strains (Fernandes et al, 2004). Furthermore, the COMT inhibitor tolcapone has been found to yield improvements in lesion- or drug-induced deficits of memory in rats (Khromova et al, 1995, 1997).

A common valine-methionine (Val/Met) substitution at codons 108 and 158 in the S-COMT and MB-COMT transcripts, respectively, has been reported to account for $>95 \%$ of the variations of this enzyme activity in humans. The Val108 allele has three- to four-fold higher enzyme activity than the Met108 allele and heterozygotes have intermediate enzyme activity (Lotta et al, 1995; Lachman et al, 1996; Weinshilboum et al, 1999). The Val158Met (rs4680) variant has been shown to significantly affect protein abundance and enzyme activity but not mRNA expression, suggesting that differences in protein integrity account for the difference in enzyme activity between alleles (Chen et al, 2004). Therefore, the Val108/158Met genetic polymorphism has been the subject of intense molecular epidemiologic study and has been reported to be associated with risk for a number of diseases including schizophrenia, obsessive-compulsive disorder, disordered cognitive abilities (for a review, see Bilder et al, 2004), anorexia nervosa (Frisch et al, 2001), anxiety (Enoch et al, 2003; McGrath et al, 2004), and drug abuse and alcoholism (Vandenbergh et al, 1997; Tiihonen et al, 1999; Horowitz et al, 2000; Kauhanen et al, 2000; Wang et al, 2001). However, many of these studies are controversial.

To date, only three studies have investigated a possible association between genetic variant(s) within COMT and smoking behavior. Two did not identify an association between COMT and smoking initiation, persistence or cessation (David et al, 2002), or tobacco consumption
(McKinney et al, 2000). Colilla and co-workers recently reported on an association between the COMT Val/Met variant with exsmoker status in EA females, but not in AA females (Colilla et al, 2005). Given the role of COMT in dopamine metabolism, the role of the dopamine reward pathway in $\mathrm{ND}$, and the evidence that nicotine stimulates the release of dopamine (Nisell et al, 1995; Pontieri et al, 1996), it makes this gene an attractive candidate gene for an association study with the vulnerability to $\mathrm{ND}$, as reported herein.

\section{MATERIALS AND METHODS}

\section{Participants and Smoking Phenotypes}

Participants were of either African-American (AA) or European-American (EA) origin from the Mid-South Tobacco Family (MSTF) cohort, recruited primarily from the states of Tennessee, Mississippi, and Arkansas in the U.S. during 1999-2004. Proband smokers were required to be at least 18 years of age, to have smoked tobacco for at least the last 5 years, and have consumed an average of 20 cigarettes per day for the last 12 months. Siblings and parents of a smoking proband were recruited whenever possible, regardless of their smoking status. Extensive data were collected on each participant, including demographics (e.g., sex, age, race, biological relationships, weight, height, years of education, and marital status), medical history, smoking history and current smoking behavior, and personality traits, utilizing the various questionnaires available at the NIDA Genetics Consortium Website (http://zork.wustl.edu/nida). All participants provided informed consent. The study protocol and forms/procedures were approved by all participating Institutional Review Boards.

The degree of ND of each smoker was ascertained by the three most commonly used measures: Smoking Quantity ( $\mathrm{SQ}$; defined as the number of cigarettes smoked per day), the Heaviness of Smoking Index (HSI; 0-6 scale), and the Fagerstrõm Test for ND score (FTND; 0-10 scale) (Heatherton et al, 1991). Our primary reasons for examining all three measures were: (a) the current lack of consensus as to the best approach to assess ND as a phenotype, and (b) to permit maximum cross-reference with previous studies of ND. The SQ provides a simple, quantified index of the amount of consumption (using a 0-3 point compressed format), whereas HSI includes one item addressing quantity (SQ) and another item assessing urgency, that is, 'How soon after you wake up do you smoke your first cigarette?' The FTND score includes the HSI plus other indicators of behavioral propensity to smoke under various circumstances. The FTND has been accepted as a standard in both clinical and research settings, although recent evidence suggests ND is a broader and more complex construct than previously considered (Swan, 2003). Given the presence of overlap in the content of the three ND measures, there exist fairly robust correlations among them $(r=0.88-0.94)$. Of the 2037 participants, the average age was $39.4 \pm 14.4$ (SD) years for the AAs and $40.5 \pm 15.5$ years for EAs. The average nuclear family size was $3.14 \pm 0.75$ for AAs and $3.17 \pm 0.69$ for EAs. The average HSI and FTND scores of smokers were $3.7 \pm 1.4$ and 
$6.26 \pm 2.15$ for AAs and $3.9 \pm 1.4$ and $6.33 \pm 2.22$ for EAs, respectively. The average number of cigarettes smoked per day was $19.4 \pm 13.3$ for AA smokers and $19.5 \pm 13.4$ for EA smokers. A detailed description of demographic and clinical characteristics for the participants in the study is presented in Table 1.

\section{DNA Extraction, SNP Selection, and SNP Genotyping}

DNA was extracted from peripheral blood samples using an extraction kit purchased from Qiagen Inc. (Valencia, CA). SNPs selected for our investigation include the commonly used Val/Met missense SNP (rs4680) and a SNP (rs4633) within exon 3 of the MB-COMT transcript or exon 1 of the $\mathrm{S}-C O M T$ transcript that defines a synonymous $\mathrm{C} \rightarrow \mathrm{T}$ (His/ His) change. To have a better coverage of the $27 \mathrm{~kb}$ genomic region, we also selected three intronic SNPs (Figure 1). All five SNPs investigated in this study were selected from the NCBI SNP-database (http://www.ncbi.nlm.nih.gov/entrez/ query.fcgi? $\mathrm{db}=\mathrm{snp}$ ), and had a minimum allele frequency (MAF) of 0.15 or greater according to the frequency from the database. Information regarding these SNPs, including their location within the gene, chromosomal position, allelic variants, and primer/probe sequences, is listed in Table 2.

Table I Clinical Characteristics for the Pooled, EA and AA Samples

\begin{tabular}{lccc}
\hline Characteristic & $\begin{array}{c}\text { African- } \\
\text { American }\end{array}$ & $\begin{array}{c}\text { European- } \\
\text { American }\end{array}$ & Pooled \\
\hline No. of nuclear families & 402 & 200 & 602 \\
Avg. members/family & $3.14 \pm 0.75$ & $3.17 \pm 0.69$ & $3.15 \pm 0.73$ \\
No. of subjects & 1366 & $67 \mid$ & 2037 \\
Gender (\% female) & 66.1 & 69.5 & 67.2 \\
Age (years) & $39.4 \pm \mid 4.4$ & $40.5 \pm 15.5$ & $39.7 \pm 14.8$ \\
No. of smokers & 1053 & 515 & 1568 \\
Age of smoking onset (years) & $17.3 \pm 4.7$ & $15.5 \pm 4.4$ & $16.7 \pm 4.7$ \\
Years smoked & $20.4 \pm 12.5$ & $23.2 \pm 13.5$ & $21.3 \pm 12.9$ \\
No. cigarettes/day & $19.4 \pm 13.3$ & $19.5 \pm 13.4$ & $19.5 \pm 13.3$ \\
HSI score & $3.7 \pm 1.4$ & $3.9 \pm 1.4$ & $3.8 \pm 1.4$ \\
FTND score & $6.26 \pm 2.15$ & $6.33 \pm 2.22$ & $6.29 \pm 2.17$ \\
\hline
\end{tabular}

The SNPs were genotyped using the TaqMan assay in a 384-well microplate format (Applied Biosystems Inc., Foster City, CA). Briefly, $15 \mathrm{ng}$ of DNA was amplified in a total volume of $7 \mu \mathrm{l}$ containing an MGB probe and $2.5 \mu \mathrm{l}$ of TaqMan universal PCR master mix. The amplification conditions were $2 \mathrm{~min}$ at $50^{\circ} \mathrm{C}$ and $10 \mathrm{~min}$ at $95^{\circ} \mathrm{C}$ followed by 40 cycles of $95^{\circ} \mathrm{C}$ for $25 \mathrm{~s}$ and $60^{\circ} \mathrm{C}$ for $1 \mathrm{~min}$. Allelic discrimination analysis was performed on the ABI Prism 9700 Sequence Detection System. To ensure the quality of the genotyping, consistent results were required for eight control samples added to each 384-well reaction plate.

\section{Statistical and Association Analyses}

Pair-wise LD between all SNP markers was assessed using the program Haploview (Barrett et al, 2005) with the option of determining haplotype blocks according to the criteria defined by Gabriel et al (2002). The PedCheck program (O'Connell and Weeks, 1998) was used to identify any inconsistent Mendelian inheritance, nonpaternity, or typing errors. A total of 136 genotyping inconsistencies were detected in the AA sample and 66 in the EA sample of approximately 10000 assays and were excluded from all subsequent statistical analyses.

Associations between a single SNP and the three ND measures were determined by the PBAT program using generalized estimating equations (Lange et al, 2003). Since the current version of PBAT (v. 1.2) cannot perform haplotype-based association analysis, the FBAT program was used to determine associations between each ND measure and haplotypes from multiple SNP combinations with the option of computing $P$-values of the $Z$ statistic using Monte Carlo sampling under the null distribution of no linkage and no association (Horvath et al, 2004). In the present analysis, we tested all consecutive three and four multilocus combinations, the five SNP haplotype combinations, as well as all other possible three and four SNP combinations. Gene $\times$ ethnicity interactions for the pooled sample and gene $\times$ gender interactions for the pooled, AA, and EA samples were determined by FBAT interaction statistics, an option provided within PBAT (for details, see http://www.biostat.harvard.edu/ clange/pbat3/default.htm). Three genetic models (additive, dominant, and recessive) were tested for both individual and multi-locus SNPs. For all FBAT and PBAT association tests, we applied sex and age

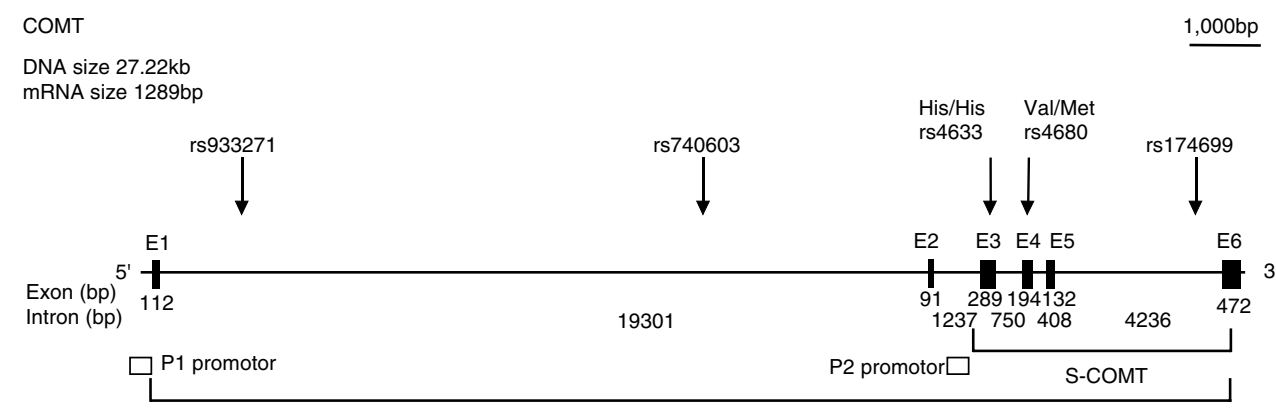

Figure I COMT gene structure and location of the five SNPs used in the present investigation. The two exonic SNPs, rs4633 and rs4680, cause a synonymous (His/His) and nonsynonymous (Val/Met) amino acid change, respectively. The two promoters (PI and P2) are indicated as well as the two isoforms, MB-COMT and S-COMT, resulting from transcription from PI and P2, respectively. 
Table 2 Positions, Nucleotide Variation, Minor Allele Frequency and Primer/Probe Sequences of 5 SNPs Within COMT

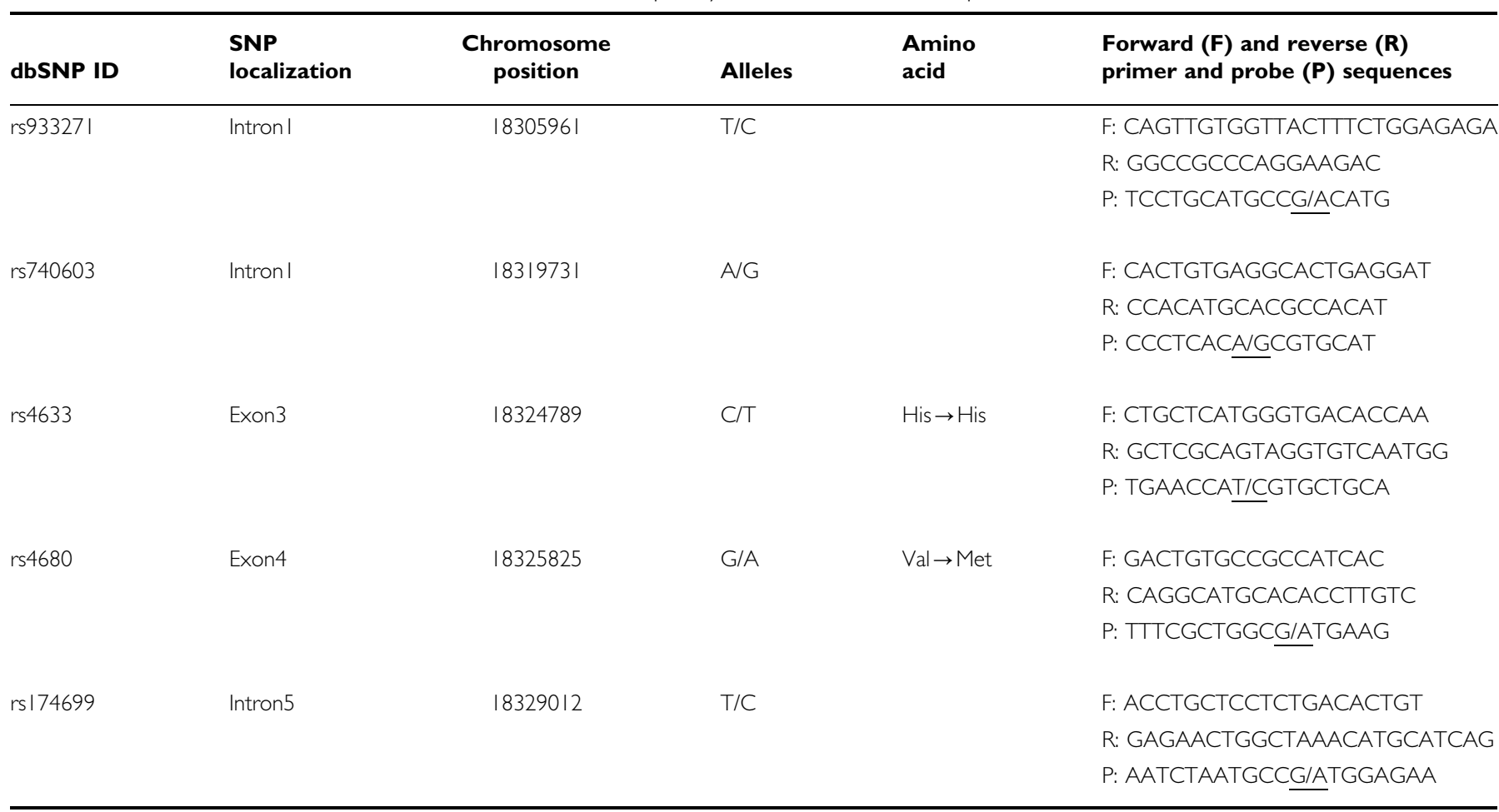

as covariates when analyzing the AA and EA samples separately; sex, age, and ethnicity covariates were employed in the pooled sample, and age as a covariate in the AA and EA samples for each gender. We used these covariates because they have been previously shown to influence ND (Edwards et al, 1995; Perez-Stable et al, 1998; Benowitz et al, 1999; Heath et al, 1999; Madden et al, 1999; Li et al, 2003). The three ND measures, SQ, HSI, and FTND, were analyzed individually. All associations found to be significant were corrected for multiple testing according to the SNP spectral decomposition (SNPSpD) approach (Nyholt, 2004) for individual SNP analysis, and using Bonferroni correction by dividing the significance level by the number of major haplotypes (frequency $>5.0 \%$ ) for haplotype-based association analysis.

\section{RESULTS}

\section{Association Analysis of Individual SNPs}

Individual SNP analysis using PBAT-GEE revealed a significant association for the Val/Met polymorphism (rs4680) with all three adjusted ND measures in the pooled samples; this association remained significant after correction for multiple testing for FTND under both the dominant $(P=0.006)$ and recessive $(P=0.007)$ models (adjusted $P$ value at the 0.05 significance level is 0.013 ; Table 3 ). Given the potential genetic differences in ND across racial groups (Benowitz et al, 1999), we used FBAT interaction statistics to determine the presence of heterogeneity in our samples and found a significant marker-ethnicity interaction for all three ND measures in the pooled samples for the Val/Met variant $(P$-value ranged between 0.003 and 0.01 for all three
ND measures; data not shown). Moreover, we compared the allele frequencies of the five SNPs within COMT in the pooled, AA and EA samples and noticed differences between the two ethnic samples for several SNPs (based on calculations of the allele frequency by directly counting the numbers of each allele from the progenitors of our samples; Table 4). This suggests an ethnic-specific allele distribution in our AA and EA samples, which is consistent with results reported by other researchers for the Val/Met variant (Palmatier et al, 1999; DeMille et al, 2002).

As a result of the significant gene and ethnicity interaction present in the pooled samples and the different distribution of the allele frequencies across ethnic samples, we analyzed the AA and EA samples separately to minimize this source of heterogeneity. Individual SNP analysis for each ethnic group resulted in a significant association for SNP rs4680 with all three age- and sex-adjusted ND measures under different genetic models in the EA sample $(P=0.009-0.02$; Table 3$)$. This association remained significant for the adjusted SQ after correcting for multiple testing. In the AA sample, we also found a significant association of rs4680 with the FTND score $(P=0.04)$. However, this association was no longer significant after correction for multiple testing (Table 3).

In addition to examining an ethnic-specific effect of COMT on ND, we also investigated gender specificity by analyzing the males and females of each ethnicity separately. Such an effort was motivated by: (1) our finding of a significant gender-gene interaction for rs4680 in the pooled and EA samples $(P=0.006-0.02)$ for the three ND measures (data not shown); and (2) reports of sex-specific enzyme activity and sexually dimorphic effects for COMT on genetic susceptibility to several psychiatric disorders (Boudikova 
Table 3 P-Values for Association of Single SNPs Within COMT with Three ND Measures in the Pooled, AA and EA Samples

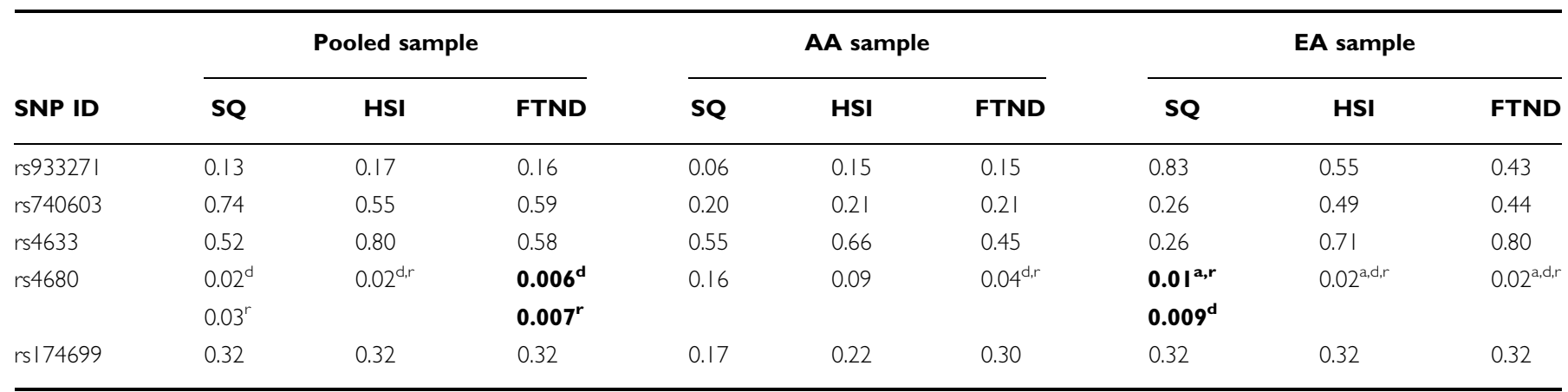

The adjusted $P$-value after correction for multiple testing at the 0.05 significance level is 0.0 l (bold).

Superscripts indicate the genetic models used for analysis; $a=$ additive, $d=$ dominant, and $r=$ recessive model.

For the pooled samples, the three ND measures were adjusted for age, sex, and ethnicity; for each ethnic-specific sample group only age and sex were used as covariates for the ND measures.

Table 4 Observed Versus Reported Allele Frequency of Five SNPs Within COMT

\begin{tabular}{llccc}
\hline dbSNP ID & $\begin{array}{c}\text { Major/minor } \\
\text { alleles }\end{array}$ & $\begin{array}{c}\text { Corresponding minor allele } \\
\text { frequency in pooled } \\
\text { samples }\end{array}$ & $\begin{array}{c}\text { Corresponding minor } \\
\text { allele frequency in AA } \\
\text { samples }\end{array}$ & $\begin{array}{c}\text { Corresponding minor } \\
\text { allele frequency in EA } \\
\text { samples }\end{array}$ \\
\hline rs93327I & T/C & 0.33 & 0.39 & 0.42 \\
rs740603 & A $/ G$ & 0.47 & 0.32 & 0.53 \\
rs4633 & G/T & 0.41 & 0.48 & 0.52 \\
rs4680 & G/A & 0.59 & 0.02 & 0.73 \\
rs174699 & T/C & 0.03 & & 0.54 \\
\hline
\end{tabular}

et al, 1990; Gogos et al, 1998; Weinshilboum et al, 1999; Chen et al, 2004). We found a nominally significant association of rs 4680 with all three ND measures in the EA males $(P=0.03-0.049)$ and with SQ in the AA females $(P=0.03-0.04)$; however, these associations were no longer significant after correction for multiple testing.

The pair-wise D' values of the five SNPs within COMT were generally low except for rs4633 and rs4680, indicating that these two SNPs are highly linked (Figure 2). According to the criteria for block definition from Gabriel and colleagues (Gabriel et al, 2002), a single haplotype block of $1.0 \mathrm{~kb}$ encompassing rs 4633 and rs 4680 could be found in the EA sample, but it was not present in the AA sample.

\section{Haplotype Analysis of Multiple SNPs}

Haplotype-based association analysis was performed for all possible three- and four-SNP combinations (including consecutive and nonconsecutive SNPs), and the five-SNP combination within COMT. Based on differences found across the ethnic samples for the single SNP analysis, we performed separate haplotype analyses on the AA and EA samples. In the AA sample, we found a major haplotype AG-T (frequency of $23.6 \%$ ) formed by the consecutive SNPs rs740603-rs4680-rs174699 (spanning $9.3 \mathrm{~kb}$ ) that revealed a significant inverse association with all three adjusted ND measures (minimum $Z=-3.35 ; P=0.0005$ for the FTND score; Table 5A). These associations remained significant after Bonferroni correction for testing of four major haplotypes for HSI and FTND under the dominant model.
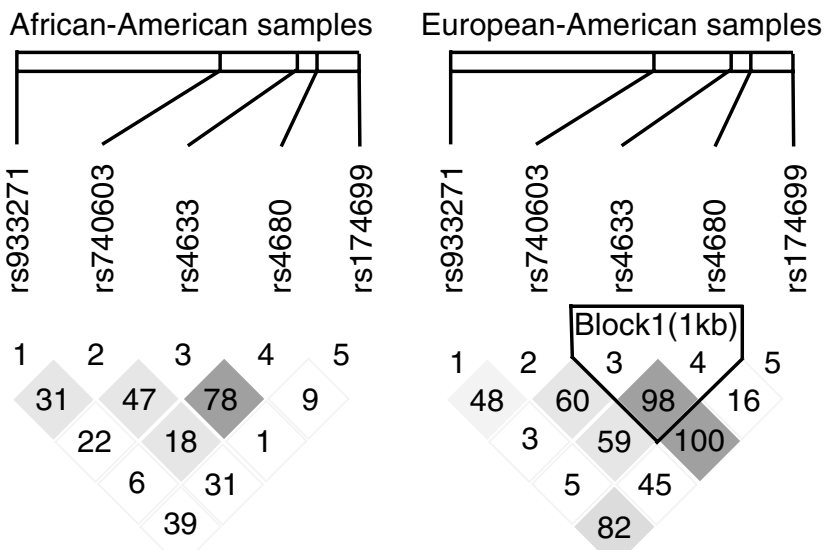

Figure 2 Haploview-generated LD map of five SNPs within COMT in the $A A$ and EA samples. Regions of high $L D\left(D^{\prime}=1\right.$ and $\left.L O D>2\right)$ are shown in dark gray. Markers with lower LD $(0.21<D<1$ and $L O D>2)$ are shown in tints of gray, with the intensity decreasing with decreased $D^{\prime}$ value. Regions of low LD and low LOD scores $(L O D<2)$ are shown in white. Haplotype blocks were defined by the Haploview program with the option of using the haplotype block definition proposed by Gabriel et al (2002).

In the EA sample, no significantly-associated major haplotypes for this three-SNP combination could be found.

Haplotype analysis of the nonconsecutive SNP combination rs933271-rs4680-rs174699 (spanning $23 \mathrm{~kb}$ ), revealed a major T-G-T haplotype at a frequency of $15.2 \%$ in the EA 
Table 5 Z and Permutation P-Values for the Significant Associated COMT Haplotypes for rs740603-rs4680-rs 174699 (A) and rs93327I-rs4680-rs 174699 (B) with Three ND Measures in the AA and EA Samples

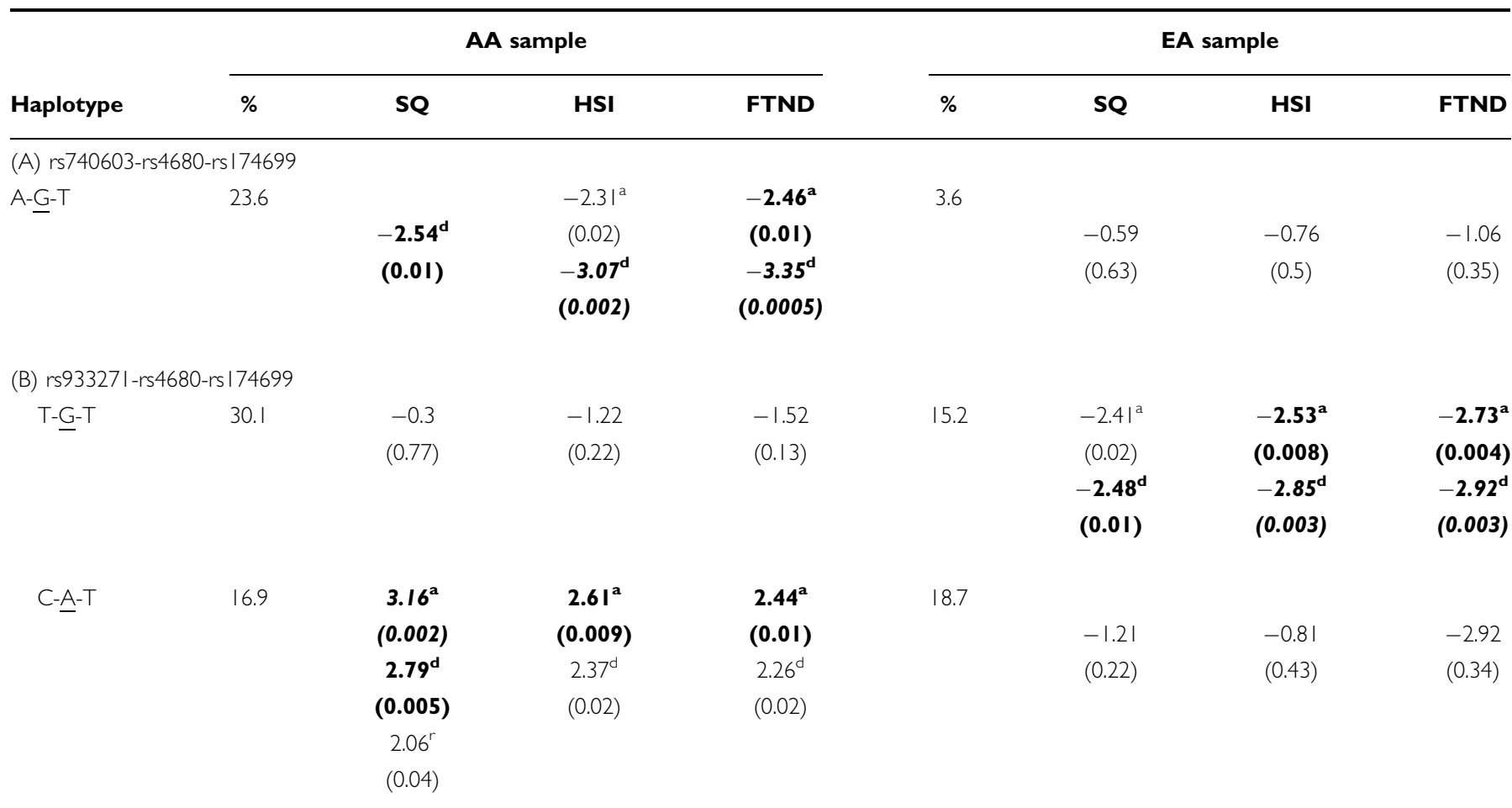

The adjusted $P$-value at the 0.05 significance level after Bonferroni correction for four major haplotypes in the AA sample is 0.0125 (bold) and at the 0.01 significance level is 0.0025 (bold and italics); for three major haplotypes in the EA sample are 0.017 (bold) and 0.003 (bold and italics), respectively.

Superscripts indicate the genetic models used in the analysis; $a=$ additive and $d=$ dominant model.

The ND measures used in the analysis were corrected for age and sex.

sample that showed a significant negative association after Bonferroni correction with all three ND measures (minimum $Z=-2.92 ; P=0.003$ for the FTND score; Table $5 \mathrm{~B}$ ). In contrast, for the AA sample, we found a significant positive association for a different haplotype C-A-T (present in $16.9 \%)$ for this three-SNP combination with all three ND measures (maximum $Z=3.16 ; \quad P=0.002$ for SQ). By examining the allele combination of each significant haplotype, we found that the high activity Val allele (underlined $\mathrm{G}$ in the haplotypes) is associated with protective haplotypes, whereas the low-activity Met allele (underlined $\mathrm{A}$ in the haplotype) is present in the high-risk haplotypes.

Similar to the analytical strategy used for individual SNPs, we examined the association between haplotypes and ND measures in males and females separately. Haplotype analysis of rs740603-rs4680-rs174699 revealed a major AG-T haplotype (frequency of $23.6 \%$ ) in the AA females that showed a significant inverse association after Bonferroni correction with HSI and FTND (minimum $\mathrm{Z}=-3.10$; $P=0.002$; Table $6 \mathrm{~A}$ ). In addition, two major haplotypes, formed by SNPs 933271-rs4680-rs174699, showed significant associations with three ND measures in the EA male sample subgroup. The first major haplotype T-A-T with a frequency of $56.7 \%$, not found to be significant in the previous analysis collapsing across gender (Table 6B), now revealed a significant positive association after Bonferroni correction with all three ND measures (maximum $Z=3.11$; $P=0.001$ for $\mathrm{SQ}$ ). A second major haplotype, T-G-T
(15.2\%), which was previously found to be significant in overall analyses, now also showed a significant negative association with all three ND measures for EA males (minimum $Z=-3.59 ; P=0.00002$ ). Finally, we found a different haplotype, C-A-T (16.9\%), formed by the same rs933271-rs4680-rs174699 SNP combination that was significantly associated with $\mathrm{SQ}$ in AA females $(Z=2.44$, $P=0.01$ ). Consistent with our previous analyses, the highactivity Val allele (underlined $G$ in the haplotypes) is associated with protective haplotypes whereas the lowactivity Met allele (underlined A in the haplotype) is present in the high-risk haplotypes in these gender-specific findings.

\section{DISCUSSION}

Dopaminergic pathways play an important role in the pathogenesis of ND. This suggests the potential importance of COMT, which has been shown to moderate dopamine metabolism via inhibitory mechanisms. We tested this hypothesis in the 602 nuclear families comprised of smokers and nonsmokers of either AA or EA origin in the MSTF cohort to determine whether behavioral associations with COMT polymorphisms exist. Two exonic SNPs, including the nonsynonymous Val/Met variant (rs4680) that has been extensively reported in the literature, and three intronic SNPs within COMT were analyzed for association with three adjusted ND measures. We 
Table 6 Z and Permutation P-Values for the Significant Associated COMT Haplotypes rs740603-rs4680-rs 174699 (A) and rs93327Irs4680-rs 174699 (B) with Three ND Measures in Males and Females of AA and EA Samples

\begin{tabular}{|c|c|c|c|c|c|c|c|c|}
\hline \multirow[b]{2}{*}{ Gender/Haplotype } & \multicolumn{4}{|c|}{ AA sample } & \multicolumn{4}{|c|}{ EA sample } \\
\hline & $\%$ & sQ & HSI & FTND & $\%$ & sQ & HSI & FTND \\
\hline \multicolumn{9}{|l|}{ Female } \\
\hline \multirow[t]{2}{*}{ A-G-G } & 23.6 & & $-2.75^{\mathrm{a}}$ & $-2.82^{\mathrm{a}}$ & 3.6 & -0.27 & -0.31 & -0.48 \\
\hline & & & $(0.003)$ & -0.002 & & & & \\
\hline \multicolumn{9}{|c|}{ (B) rs93327|-rs4680-rs I 74699} \\
\hline \multicolumn{9}{|l|}{ Male } \\
\hline \multirow[t]{3}{*}{ T-A-T } & & & & & 56.7 & $3 . I I^{\mathrm{a}}$ & $2.91^{\mathrm{a}}$ & $2.83^{\mathrm{a}}$ \\
\hline & & & & & & $(0.00 I)$ & $(0.002)$ & $(0.003)$ \\
\hline & & & & & & $(0.01)$ & $(0.01)$ & $(0.01)$ \\
\hline \multirow[t]{4}{*}{$T-\underline{G}-T$} & 30.1 & -1.24 & -1.38 & -1.49 & 15.2 & $-3.47^{\mathrm{a}}$ & $-3.50^{\mathrm{a}}$ & $-3.59^{\mathrm{a}}$ \\
\hline & & $(0.2)$ & $(0.16)$ & $(0.13)$ & & $(0.001)$ & $(0.0002)$ & $(0.00002)$ \\
\hline & & & & & & $-3.1 I^{\mathrm{d}}$ & $-3.45^{\mathrm{d}}$ & $-3.47^{\mathrm{d}}$ \\
\hline & & & & & & $(0.001)$ & $(0.00008)$ & $(0.0002)$ \\
\hline
\end{tabular}

The adjusted $P$-value at the 0.05 significance level after Bonferroni correction for four major haplotypes in the AA sample is 0.0 I 25 (bold) and at the 0.01 significance level is 0.0025 (bold and italics); for three major haplotypes in the EA sample are 0.017 (bold) and 0.003 (bold and italics), respectively.

Superscripts indicate the genetic models used in the analysis; $a=$ additive, $d=$ dominant, and $r=$ recessive model.

The ND measures were corrected for age.

performed association analysis on the pooled sample and the two ethnic groups separately. The reasons for this are three-fold. First, the frequencies of the Val and Met alleles are substantially different among several ethnic populations. The low-activity allele (Met) is less common in individuals of African origin (McLeod et al, 1994; Palmatier et al, 1999; Ameyaw et al, 2000; DeMille et al, 2002), and multimarker haplotypes in COMT show marked differences across populations (Palmatier et al, 2004). These findings are consistent with the findings of the current study in which the A (Met) allele was present in $48 \%$ in the AA sample and $73 \%$ in the EA sample. Further, our ethnic samples showed a different haplotype block structure within COMT. Second, ethnic differences in nicotine metabolism (Perez-Stable et al, 1998; Benowitz et al, 1999) and gender differences, both in the response to smoking (Perkins et al, 1999) and in the genetic influences on ND (Li et al, 2003) have been reported. Although it remains to be established (Pearce et al, 2004) evidence suggests that there are genetic differences among ethnic groups (Burchard et al, 2003; Bamshad et al, 2004). And third, we found a significant marker and ethnicity interaction for rs4680 in our pooled sample.

After correction for multiple testing, significant associations of the $\mathrm{Val} /$ Met variant (rs4680) within COMT were found in the pooled and EA samples for the FTND score and SQ, respectively. Haplotype analysis of three-SNP combinations with $\mathrm{ND}$, including the Val/Met variant, revealed a major A-G-T protective haplotype for rs740603-rs4680rs174699 in the AA sample and a major T-G-T protective haplotype in the EA sample for rs933271-rs4680-rs174699. This latter three-SNP combination also showed a different major C-A-T haplotype in the AA sample that was positively associate with the three ND measures. Our results indicate that an ethnic-specific haplotype within the COMT gene is associated with $\mathrm{ND}$, a finding consistent with the ethnic differences in allele frequencies of some of the SNPs examined, and with the LD/block structure differences between the two ethnic groups. The protective haplotypes have the Val ( $\underline{G}$ in the haplotype) allele whereas the highrisk haplotypes contain the Met (A in the haplotype) allele in all three associated haplotypes. This suggests that in our sample, the low enzyme activity Met allele correlates with a higher vulnerability to develop ND. Recently, Colilla et al (2005) found overtransmission of the Met allele in female exsmokers suggesting that the Met allele is correlated with successful quitting whereas in our results the Met allele is associated with ND. Our results are in line with findings from both animal and human studies which show that reward processes are mediated by dopaminergic pathways from the ventral tegmental area to nucleus accumbens and frontal cortex (Kalivas, 1993), and that nicotine stimulates the release of dopamine from neurons (Corrigall et al, 1992; Nisell et al, 1994; Pontieri et al, 1996; Balfour et al, 2000; Smolka et al, 2004). Therefore, individuals with low-activity COMT haplotypes may experience a longer-lasting and 
more effective dopamine release in the brain, thereby increasing the magnitude and/or duration of reward derived from smoking and the risk of becoming nicotine dependent.

Several lines of evidence suggest that COMT activity differs between men and women, most likely due to the effects of estrogen metabolism. It has been shown that COMT expression is regulated by estrogen through the estrogen-responsive elements in its promoter region (Xie et al, 1999). Women generally have $20-30 \%$ lower COMT activity levels than men (Boudikova et al, 1990). In addition, sexually dimorphic effects of COMT have been shown in COMT-mutant mice (Gogos et al, 1998), and in genetic susceptibility to affective disorder, obsessive-compulsive disorder, anxiety and panic disorder, and schizophrenia (Karayiorgou et al, 1997; Shifman et al, 2002; Enoch et al, 2003; Lee et al, 2005). Our findings suggest a sex-specific genetic component of ND; in particular, identification of the protective A-G-T haplotype of rs740603-rs4680-rs174699 in AA females, and the protective T-G-T haplotype of rs933271-rs4680-rs174699 in EA males.

It has been suggested that the effect of the Val/Met polymorphism on dopamine-mediated frontal cortical function may be the neurobiological mechanism underlying the clinical association with psychological disorders (Weinberger et al, 2001). This polymorphism has been linked to various diseases/disorders, including schizophrenia, Parkinson's disease, obsessive-compulsive disorder, drug abuse and alcoholism, albeit the associations are generally weak and replication has been inconsistent. Thus, it is difficult to conclude unequivocally whether the risk of any of these disorders is influenced by COMT. Some of the inconsistency could be attributable to: (1) population-substructure differences between different study samples; (2) definition of the complex traits analyzed; and (3) lack of power to detect an association due to small sample sizes or SNP selection. In this study, we considered these shortcomings and addressed them to the degree possible. Since our sample is family-based, we minimized or excluded potential confounding effects of population stratification. Furthermore, we used a large sample set of extensively phenotyped smokers and nonsmokers of two ethnicities, which provides increased power to detect associations between genetic variants and ND. The three measures analyzed are those most commonly used in genetic research and clinical settings to assess ND, permitting the opportunity for cross-study replication by others.

We included additional SNPs in our analysis besides the Val/Met variant and performed both single-SNP and haplotype analyses. We observed additional significant results for haplotype analysis as compared with single SNP analysis. These data suggest that if the COMT gene is indeed involved in susceptibility to ND, its influence cannot be fully accounted for by the Val/Met polymorphism, and other functional variants close to or in strong LD with these SNPs are likely to exist. In that case, COMT may be a modifier of the smoking phenotype, rather than a direct susceptibility allele. Moreover, since the LD between the analyzed SNPs was generally low, we plan to analyze more SNPs within COMT, as well as adjacent genes. Although the $\mathrm{Val} / \mathrm{Met}$ variant of the COMT gene has been extensively studied, the underlying mechanism remains unknown (Goodman et al, 2002) and we can only speculate on the nature of the protective effect of the Val allele in our smoker sample.

In summary, we provide strong evidence for a relationship between allelic variants of COMT and three ND measures in EA and AA smokers. We found ethnic-specific protective haplotypes within $C O M T$ against the expression of ND, as well as gender differences of COMT haplotypes in both AA and EA populations. Finally, regardless of ethnicity and gender, the high enzyme activity Val allele is always present in the protective haplotypes, whereas the low activity Met allele is associated with high-risk haplotypes for ND.

\section{ACKNOWLEDGEMENTS}

We acknowledge the invaluable contributions of all participants who completed questionnaires and provided blood samples for the study. The clinical and research staff at The University of Texas Health Science Center at San Antonio, University of Tennessee Health Science Center, and the ACT Center of the University of Mississippi Medical Center have been involved in clinical recruitment and genetic analysis, and we thank them for their dedicated work. This project is funded by a grant from the National Institute on Drug Abuse to Ming D Li (DA-12844) and by general support for Thomas Payne via a grant from The Partnership for a Healthy Mississippi to the University of Mississippi School of Dentistry.

\section{REFERENCES}

Akil M, Kolachana BS, Rothmond DA, Hyde TM, Weinberger DR, Kleinman JE (2003). Catechol-O-methyltransferase genotype and dopamine regulation in the human brain. J Neurosci 23: 2008-2013.

Ameyaw MM, Syvanen AC, Ulmanen I, Ofori-Adjei D, McLeod HL (2000). Pharmacogenetics of catechol-O-methyltransferase: frequency of low activity allele in a Ghanaian population. Hum Mutat 16: 445-446.

Balfour DJ, Wright AE, Benwell ME, Birrell CE (2000). The putative role of extra-synaptic mesolimbic dopamine in the neurobiology of nicotine dependence. Behav Brain Res 113: 73-83.

Bamshad M, Wooding S, Salisbury BA, Stephens JC (2004). Deconstructing the relationship between genetics and race. Nat Rev Genet 5: 598-609.

Barrett JC, Fry B, Maller J, Daly MJ (2005). Haploview: analysis and visualization of LD and haplotype maps. Bioinformatics 21: 263-265.

Benowitz NL, Perez-Stable EJ, Fong I, Modin G, Herrera B, Jacob III P (1999). Ethnic differences in $N$-glucuronidation of nicotine and cotinine. J Pharmacol Exp Ther 291: 1196-1203.

Bilder RM, Volavka J, Lachman HM, Grace AA (2004). The catechol-O-methyltransferase polymorphism: relations to the tonic-phasic dopamine hypothesis and neuropsychiatric phenotypes. Neuropsychopharmacology 29: 1943-1961.

Boudikova B, Szumlanski C, Maidak B, Weinshilboum R (1990). Human liver catechol-O-methyltransferase pharmacogenetics. Clin Pharmacol Ther 48: 381-389.

Burchard EG, Ziv E, Coyle N, Gomez SL, Tang H, Karter AJ et al (2003). The importance of race and ethnic background in biomedical research and clinical practice. $N$ Engl J Med 348: 1170-1175.

Chen J, Lipska BK, Halim N, Ma QD, Matsumoto M, Melhem S et al (2004). Functional analysis of genetic variation in catechol-O- 
methyltransferase (COMT): effects on mRNA, protein, and enzyme activity in postmortem human brain. Am J Hum Genet 75: 807-821.

Colilla S, Lerman C, Shields PG, Jepson C, Rukstalis M, Berlin J et al (2005). Association of catechol-O-methyltransferase with smoking cessation in two independent studies of women. Pharmacogenet Genomics 15: 393-398.

Corrigall WA, Franklin KB, Coen KM, Clarke PB (1992). The mesolimbic dopaminergic system is implicated in the reinforcing effects of nicotine. Psychopharmacology (Berlin) 107: 285-289.

David SP, Johnstone E, Griffiths SE, Murphy M, Yudkin P, Mant D et al (2002). No association between functional catechol Omethyl transferase $1947 \mathrm{~A}>\mathrm{G}$ polymorphism and smoking initiation, persistent smoking or smoking cessation. Pharmacogenetics 12: 265-268.

DeMille MM, Kidd JR, Ruggeri V, Palmatier MA, Goldman D, Odunsi A et al (2002). Population variation in linkage disequilibrium across the COMT gene considering promoter region and coding region variation. Hum Genet 111: 521-537.

Edwards KL, Austin MA, Jarvik GP (1995). Evidence for genetic influences on smoking in adult women twins. Clin Genet 47: 236-244.

Enoch MA, Xu K, Ferro E, Harris CR, Goldman D (2003). Genetic origins of anxiety in women: a role for a functional catechol-Omethyltransferase polymorphism. Psychiatr Genet 13: 33-41.

Fernandes C, Paya-Cano JL, Sluyter F, D'Souza U, Plomin R, Schalkwyk LC (2004). Hippocampal gene expression profiling across eight mouse inbred strains: towards understanding the molecular basis for behaviour. Eur J Neurosci 19: 2576-2582.

Frisch A, Laufer N, Danziger Y, Michaelovsky E, Leor S, Carel C et al (2001). Association of anorexia nervosa with the high activity allele of the COMT gene: a family-based study in Israeli patients. Mol Psychiatry 6: 243-245.

Gabriel SB, Schaffner SF, Nguyen H, Moore JM, Roy J, Blumenstiel $B$ et al (2002). The structure of haplotype blocks in the human genome. Science 296: 2225-2229.

Garris PA, Collins LB, Jones SR, Wightman RM (1993). Evoked extracellular dopamine in vivo in the medial prefrontal cortex. J Neurochem 61: 637-647.

Gogos JA, Morgan M, Luine V, Santha M, Ogawa S, Pfaff D et al (1998). Catechol-O-methyltransferase-deficient mice exhibit sexually dimorphic changes in catecholamine levels and behavior. Proc Natl Acad Sci USA 95: 9991-9996.

Goodman JE, Jensen LT, He P, Yager JD (2002). Characterization of human soluble high and low activity catechol-O-methyltransferase catalyzed catechol estrogen methylation. Pharmacogenetics 12: $517-528$.

Grossman MH, Emanuel BS, Budarf ML (1992). Chromosomal mapping of the human catechol-O-methyltransferase gene to 22q11.1-q11.2. Genomics 12: 822-825.

Hatchell PC, Collins AC (1980). The influence of genotype and sex on behavioral sensitivity to nicotine in mice. Psychopharmacology (Berlin) 71: 45-49.

Heath AC, Kirk KM, Meyer JM, Martin NG (1999). Genetic and social determinants of initiation and age at onset of smoking in Australian twins. Behav Genet 29: 395-407.

Heatherton TF, Kozlowski LT, Frecker RC, Fagerstrom KO (1991). The Fagerstrom Test for nicotine dependence: a revision of the Fagerstrom tolerance questionnaire. $\mathrm{Br} J$ Addict 86: $1119-1127$.

Holmes A (2001). Targeted gene mutation approaches to the study of anxiety-like behavior in mice. Neurosci Biobehav Rev 25: 261-273.

Horowitz R, Kotler M, Shufman E, Aharoni S, Kremer I, Cohen H et al (2000). Confirmation of an excess of the high enzyme activity COMT val allele in heroin addicts in a family-based haplotype relative risk study. Am J Med Genet 96: 599-603.
Horvath S, Xu X, Lake SL, Silverman EK, Weiss ST, Laird NM (2004). Family-based tests for associating haplotypes with general phenotype data: application to asthma genetics. Genet Epidemiol 26: 61-69.

Kalivas PW (1993). Neurotransmitter regulation of dopamine neurons in the ventral tegmental area. Brain Res Brain Res Rev 18: 75-113.

Karayiorgou M, Altemus M, Galke BL, Goldman D, Murphy DL, Ott J et al (1997). Genotype determining low catechol-Omethyltransferase activity as a risk factor for obsessivecompulsive disorder. Proc Natl Acad Sci USA 94: 4572-4575.

Kauhanen J, Hallikainen T, Tuomainen TP, Koulu M, Karvonen MK, Salonen JT et al (2000). Association between the functional polymorphism of catechol-O-methyltransferase gene and alcohol consumption among social drinkers. Alcohol Clin Exp Res 24: 135-139.

Khromova I, Rauhala P, Zolotov N, Mannisto PT (1995). Tolcapone, an inhibitor of catechol-O-methyltransferase, counteracts memory deficits caused by bilateral cholinotoxin lesions of the basal nuclei of Meynert. Neuroreport 6: 1219-1222.

Khromova I, Voronina T, Kraineva VA, Zolotov N, Mannisto PT (1997). Effects of selective catechol-O-methyltransferase inhibitors on single-trial passive avoidance retention in male rats. Behav Brain Res 86: 49-57.

Lachman HM, Papolos DF, Saito T, Yu YM, Szumlanski CL, Weinshilboum RM (1996). Human catechol-O-methyltransferase pharmacogenetics: description of a functional polymorphism and its potential application to neuropsychiatric disorders. Pharmacogenetics 6: 243-250.

Lange C, Silverman EK, Xu X, Weiss ST, Laird NM (2003). A multivariate family-based association test using generalized estimating equations: FBAT-GEE. Biostatistics 4: 195-206.

Lee SG, Joo Y, Kim B, Chung S, Kim HL, Lee I et al (2005). Association of Ala72Ser polymorphism with COMT enzyme activity and the risk of schizophrenia in Koreans. Hum Genet 116: 319-328.

Li MD, Cheng R, Ma JZ, Swan GE (2003). A meta-analysis of estimated genetic and environmental effects on smoking behavior in male and female adult twins. Addiction 98: 23-31.

Li MD, Ma JZ, Beuten J (2004). Progress in searching for susceptibility loci and genes for smoking-related behaviour. Clin Genet 66: 382-392.

Lotta T, Vidgren J, Tilgmann C, Ulmanen I, Melen K, Julkunen I et al (1995). Kinetics of human soluble and membrane-bound catechol-O-methyltransferase: a revised mechanism and description of the thermolabile variant of the enzyme. Biochemistry 34: $4202-4210$.

Lundstrom K, Salminen M, Jalanko A, Savolainen R, Ulmanen I (1991). Cloning and characterization of human placental catechol-O-methyltransferase cDNA. DNA Cell Biol 10: 181-189. Lundstrom K, Tenhunen J, Tilgmann C, Karhunen T, Panula P, Ulmanen I (1995). Cloning, expression and structure of catecholO-methyltransferase. Biochim Biophys Acta 1251: 1-10.

Madden PA, Heath AC, Pedersen NL, Kaprio J, Koskenvuo MJ, Martin NG (1999). The genetics of smoking persistence in men and women: a multicultural study. Behav Genet 29: 423-431.

Matsumoto M, Weickert CS, Beltaifa S, Kolachana B, Chen J, Hyde TM et al (2003). Catechol O-methyltransferase (COMT) mRNA expression in the dorsolateral prefrontal cortex of patients with schizophrenia. Neuropsychopharmacology 28: $1521-1530$.

McGrath M, Kawachi I, Ascherio A, Colditz GA, Hunter DJ, De Vivo I (2004). Association between catechol-O-methyltransferase and phobic anxiety. Am J Psychiatry 161: 1703-1705.

McKinney EF, Walton RT, Yudkin P, Fuller A, Haldar NA, Mant D et al (2000). Association between polymorphisms in dopamine metabolic enzymes and tobacco consumption in smokers. Pharmacogenetics 10: 483-491. 
McLeod HL, Fang L, Luo X, Scott EP, Evans WE (1994). Ethnic differences in erythrocyte catechol- $O$-methyltransferase activity in black and white Americans. J Pharmacol Exp Ther 270: 26-29.

Nisell M, Nomikos GG, Svensson TH (1994). Systemic nicotineinduced dopamine release in the rat nucleus accumbens is regulated by nicotinic receptors in the ventral tegmental area. Synapse 16: 36-44.

Nisell M, Nomikos GG, Svensson TH (1995). Nicotine dependence, midbrain dopamine systems and psychiatric disorders. Pharmacol Toxicol 76: 157-162.

Nyholt DR (2004). A simple correction for multiple testing for single-nucleotide polymorphisms in linkage disequilibrium with each other. Am J Hum Genet 74: 765-769.

O'Connell JR, Weeks DE (1998). PedCheck: a program for identification of genotype incompatibilities in linkage analysis. Am J Hum Genet 63: 259-266.

Palmatier MA, Kang AM, Kidd KK (1999). Global variation in the frequencies of functionally different catechol- $O$-methyltransferase alleles. Biol Psychiatry 46: 557-567.

Palmatier MA, Pakstis AJ, Speed W, Paschou P, Goldman D, Odunsi A et al (2004). COMT haplotypes suggest P2 promoter region relevance for schizophrenia. Mol Psychiatry 9: 859-870.

Pearce N, Foliaki S, Sporle A, Cunningham C (2004). Genetics, race, ethnicity, and health. Bmj 328: 1070-1072.

Perez-Stable EJ, Herrera B, Jacob 3rd P, Benowitz NL (1998). Nicotine metabolism and intake in black and white smokers. Jama 280: 152-156.

Perkins KA, Donny E, Caggiula AR (1999). Sex differences in nicotine effects and self-administration: review of human and animal evidence. Nicotine Tob Res 1: 301-315.

Pontieri FE, Tanda G, Orzi F, Di Chiara G (1996). Effects of nicotine on the nucleus accumbens and similarity to those of addictive drugs. Nature 382: 255-257.

Robinson SF, Marks MJ, Collins AC (1996). Inbred mouse strains vary in oral self-selection of nicotine. Psychopharmacology (Berlin) 124: 332-339.

Shifman S, Bronstein M, Sternfeld M, Pisante-Shalom A, LevLehman E, Weizman A et al (2002). A highly significant association between a COMT haplotype and schizophrenia. Am J Hum Genet 71: 1296-1302.
Smolka MN, Budde H, Karow AC, Schmidt LG (2004). Neuroendocrinological and neuropsychological correlates of dopaminergic function in nicotine dependence. Psychopharmacology (Berlin) 175: 374-381.

Sullivan PF, Kendler KS (1999). The genetic epidemiology of smoking. Nicotine Tob Res 1(Suppl 2): S51-S57; discussion S69-70.

Swan GE (2003). The need for dissemination of evidence-based results from research on nicotine and tobacco. Nicotine Tob Res 5: 7-8.

Tenhunen J, Salminen M, Lundstrom K, Kiviluoto T, Savolainen R, Ulmanen I (1994). Genomic organization of the human catechol$O$-methyltransferase gene and its expression from two distinct promoters. Eur J Biochem 223: 1049-1059.

Tiihonen J, Hallikainen T, Lachman H, Saito T, Volavka J, Kauhanen J et al (1999). Association between the functional variant of the catechol-O-methyltransferase (COMT) gene and type 1 alcoholism. Mol Psychiatry 4: 286-289.

US Department of Health and Human Services (1988). The Health Consequences of Smoking: Nicotine Addiction: A report of the Surgeon General.

Vandenbergh DJ, Rodriguez LA, Miller IT, Uhl GR, Lachman HM (1997). High-activity catechol-O-methyltransferase allele is more prevalent in polysubstance abusers. Am J Med Genet 74: 439-442.

Wang T, Franke P, Neidt H, Cichon S, Knapp M, Lichtermann D et al (2001). Association study of the low-activity allele of catechol-O-methyltransferase and alcoholism using a familybased approach. Mol Psychiatry 6: 109-111.

Weinberger DR, Egan MF, Bertolino A, Callicott JH, Mattay VS, Lipska BK et al (2001). Prefrontal neurons and the genetics of schizophrenia. Biol Psychiatry 50: 825-844.

Weinshilboum RM, Otterness DM, Szumlanski CL (1999). Methylation pharmacogenetics: catechol-O-methyltransferase, thiopurine methyltransferase, and histamine $N$-methyltransferase. Annu Rev Pharmacol Toxicol 39: 19-52.

WHO (2005). The World Health Report 2005.

Xie T, Ho SL, Ramsden D (1999). Characterization and implications of estrogenic down-regulation of human catechol- $O$ methyltransferase gene transcription. Mol Pharmacol 56: 31-38. 